\title{
Effect of prematurity on genome wide methylation in the placenta
}

\author{
Jessica Schuster ${ }^{1}$, Alper Uzun ${ }^{2}$, Joan Stablia ${ }^{1}$, Christoph Schorl ${ }^{3}$, Mari Mori ${ }^{4}$ and James F. Padbury ${ }^{2,5^{*}}$ (D)
}

\begin{abstract}
Background: Preterm birth is a significant clinical problem and an enormous burden on society, affecting one in eight pregnant women and their newborns. Despite decades of research, the molecular mechanism underlying its pathogenesis remains unclear. Many studies have shown that preterm birth is associated with health risks across the later life course. The "fetal origins" hypothesis postulates that adverse intrauterine exposures are associated with later disease susceptibility. Our recent studies have focused on the placental epigenome at term. We extended these studies to genome-wide placental DNA methylation across a wide range of gestational ages. We applied methylation dependent immunoprecipitation/DNA sequencing (MeDIP-seq) to 9 placentas with gestational age from 25 weeks to term to identify differentially methylated regions (DMRs).

Results: Enrichment analysis revealed 427 DMRs with nominally significant differences in methylation between preterm and term placentas $(p<0.01)$ and 21 statistically significant DMRs after multiple comparison correction (FDR $p<0.05$ ), of which $62 \%$ were hypo-methylated in preterm placentas vs term placentas. The majority of DMRs were in distal intergenic regions and introns. Significantly enriched pathways identified by Ingenuity Pathway Analysis (IPA) included Citrulline-Nitric Oxide Cycle and Fcy Receptor Mediated Phagocytosis in macrophages. The DMR gene set overlapped placental gene expression data, genes and pathways associated evolutionarily with preterm birth.
\end{abstract}

Conclusion: These studies form the basis for future studies on the epigenetics of preterm birth, "fetal programming" and the impact of environment exposures on this important clinical challenge.

Keywords: Preterm birth, Fetal programming, Epigenetics, Placenta

\section{Background}

Despite decades of research, the underlying cause of preterm birth remains enigmatic. It is a leading cause of newborn morbidity, hospitalization, and developmental delays [1]. In addition, preterm birth is associated with health risks across the later life course of the newborn, including cardiovascular disease, metabolic syndromes, psychiatric conditions, obesity and cognitive disabilities $[1,2]$. The "fetal origins" or Developmental Origins and Health and Disease (DOHaD) hypothesis, developed from a series of epidemiologic observations, demonstrated that measures of birth size were associated with long-term chronic

\footnotetext{
* Correspondence: jpadbury@wihri.org

${ }^{2}$ Pediatrics, Center for Computational Molecular Biology, Brown Medical

School, Brown University, Providence, Rhode Island 02906, USA

${ }^{5}$ Providence, USA

Full list of author information is available at the end of the article
}

disease risk [3]. Numerous investigations have shown that antenatal maternal environmental factors, including diet, xenobiotic exposure, stress, and lifestyle factors can alter fetal growth and result in permanent biological and physiologic changes of the offspring [3]. Environmental factors like race, diet, smoking, socioeconomic status may also increase the risk of spontaneous preterm birth $[1,4,5]$ and are associated with epigenetic alterations [6].

DNA methylation is the most well studied epigenetic mechanism of gene regulation, often associated with transcriptional silencing of downstream gene(s). The presence of the methyl group(s) alone is not sufficient for transcriptional silencing, but instead alters recruitment of component proteins related to gene repression and results in a silenced chromatin conformation. DNA methylation is an essential epigenetic mechanism in fetal development [7]. 
The placenta facilitates the exchange of gas, nutrients, and waste between the mother and the fetus, and modulates effects on the fetus from the mother's immune system, thus playing an essential role in fetal growth and development. It is also essential in understanding the long-term effects of in-utero development on post-natal disease. The placenta undergoes many changes throughout gestation and the mechanisms behind these changes need to be better understood. In an attempt to do so, several studies have examined genome wide expression differences in placentas at different time points during gestation, comparing first, second and third trimester placental methylation $[8,9]$. Changes in expression with increasing gestational age were found in common between the studies. Others are attempting to better understand placental development and fetal programming through the study of epigenetic factors, including DNA methylation of placental tissue and umbilical cord blood. Studies of umbilical cord blood from preterm and term pregnancies have releaved differences in methylation associated with gestational age $[10,11]$. Novakovic et al. have studied genome scale placental promoter methylation from the three trimesters of pregnancy, revealing a progressive increase in methylation from first to third trimester. They also identified increased inter-individual variability in third trimester samples [12]. Other studies have alsofound varied methylation differences associated with gestational age comparing placentas in the third trimester, as well as a global increase in methylation with gestational age (28-40 weeks) [13-15]. In addition, the placenta has the highest overall variability in DNA methylation when compared to other tissues [16]. These studies all support the emerging paradigm that the placenta is an active mediator of fetal well-being and neurodevelopmental outcome and can serve as a blueprint for intrauterine life [17]. This is an exploratory study seeking to investigate genome wide placental DNA methylation across a wide range of preterm gestational ages and compared it to that of placenta from term deliveries. In order to generate genome-wide information, we employed immunoprecipitation of methylated DNA followed by whole-genome sequencing, so called MeDIP-seq [18]. We hypothesize that using this approach, we would be able to identify potential regions of interest and pathways involved in and influenced by changes in placental methylation associated with preterm birth and gestational age. Our objectives were to demonstrate the feasibility of this approach and to generate placental methylation data that would be useful to our own studies and to those of others.

\section{Results}

Placental sample and patient characteristics

Placental samples of villous parenchyma were taken from four quadrants between the chorionic and basal plate. Table 1 shows summary clinical characteristics of the cohort of placental samples and the associated patients. Placental samples were obtained from six preterm pregnancies (gestational age 25-34 weeks) and three term pregnancies (37-41 weeks). The average birth weights of the fetuses were $1541 \mathrm{~g}$ vs $3033 \mathrm{~g}$ and the average gestational ages were 30 weeks vs 39 weeks, respectively. We also recorded maternal pregnancy factors including BMI, but the variance was large and thus the means were not significantly different between the two groups. All fetuses had birth weights that were appropriate for gestational age. Among placentas from the preterm pregnancies, two of the mothers were diagnosed with some degree of hypertension. There was no history of drug use. One mother, who delivered preterm, admitted to smoking during pregnancy. Detailed clinical data for each sample can be found in Additional file 1 .

\section{Differentially methylated regions (DMR) associated with preterm birth}

We used the bioinformatics tools DiffBind and DESeq2 to test for association with preterm birth using methylation peak counts as the outcome and PTB status as the independent variable. The raw zipped fastq files and the peak count matrix have all be uploaded to GEO and can be found with the following accession number: GSE120458 (https://www.ncbi.nlm.nih.gov/geo/query/acc. $c g i ? a c c=G S E 120458)$. We found 427 peaks with nominally significant differences in methylation between cases and controls, $(p<0.01)$ [see Additional file 2]. Following FDR correction, there were 21 DMRs that associate with PTB using a filter for low mean counts to maximize the number of FDR significant peaks at an adjusted $p<0.05$. These 21 significant DMRs and their annotations are shown in Table 2. The peak heights (read counts) of the 21 DMRs associated with PTB are also visualized in a heat map in which unsupervised clustering was used to group the patients (columns) (Fig. 1). The three term patients (Samples 2, 8 and 9) cluster together and are distinct from the 6 preterm samples. Among the 21 DMRs associated with PTB, $62 \%$ were hypo-methylated in preterm placentas compared to term placentas. Similar percentages were found for the uncorrected significant DMRs. We next used the $\mathrm{R}$ Bioconductor package CHipSeeker [19] to annotate the DMRs associated with PTB with their nearest gene. The 427 regions are associated to 342 unique genes. The highest percentage of DMRs map to distal intergenic regions (57.38\%) followed by introns, other than the first intron and promoter regions. A larger percentage of DMRs were located in 
Table 1 Summary of Clinical characteristics of sampled patients

\begin{tabular}{llll}
\hline Clinical Data & Cases $(n=6)$ & Controls $(n=3)$ & $P$-value \\
\hline Gestational Age (avg weeks) & 30 & 39 & 0.001 \\
Maternal BMl (avg kg) & 29.5 & 40.7 & 0.32 \\
Birth Weight (avg g) & 1541 & 3033 & 0.001 \\
Drug Use (number of samples) & 0 & 0 & - \\
$\begin{array}{l}\text { Smoking (number of samples) } \\
\text { Preeclampsia (number of samples) }\end{array}$ & 1 & 0 & - \\
$\begin{array}{l}\text { Male Sex of Infant (number of } \\
\text { samples) }\end{array}$ & 3 & 0 & - \\
$\begin{array}{l}\text { Race/Ethnicity (number of } \\
\text { samples) }\end{array}$ & White (5) Other & White (2) Hispanic & - \\
\hline
\end{tabular}

proximal promoter regions $(<1 \mathrm{~kb}$ upstream) compared to more distal regions ( $>2-3 \mathrm{~kb}$ followed by $1-2 \mathrm{~kb}$ upstream) (see Additional file 3).

Enrichment scores for a variety of genomic features for the hyper-methylated and the hypo-methylated DMRs independently are shown in Fig. 2. The hypomethylated DMRs were enriched for CpG Islands and the hyper-methylated regions were enriched for $\mathrm{CpG}$ shores and shelves.

\section{Differentially methylated regions (DMR) are associated with gestational age}

We used the same pipeline to test for association of DNA methylation with preterm birth using methylation peak counts as the outcome and gestational age in weeks as a continuous, independent variable. We found 667 peaks with nominally significant differences in methylation between cases and controls, $(p<0.01)$ [see Additional file 4]. Following FDR correction, we found 67 significant DMRs that associate with gestational age, using a filter for low mean counts to maximize the number of FDR significant peaks at an adjusted $p<0.05$. Table 3 contains these 67 DMR and their annotations. The percentages of the DMRs that map to the genomic annotation categories are almost identical to the comparison between preterm birth and term.

In an attempt to distinguish DMRs that are solely a result of gestational timing from those which could be explained by experience dependent alterations, we looked for overlap and differences between the continuous analysis on gestation age and the categorical analysis on PTB status. Ten out of the 21 DMRs show methylation changes that are associated to both PTB and gestational age. The remaining 11 DMRs may reflect changes due to experience dependent alterations. Additionally, 215 DMRs were found significant in both the dichotomous and continuous models ( $p$-value <.01), mapping to 177 unique genes.

\section{Comparative gene set analysis}

To enhance discovery and interpretation of these findings, we compared our DMRs and their nearest annotated genes with previously established gene sets that

Table 2 Annotated DMR's Associated with Preterm Birth

\begin{tabular}{|c|c|c|c|c|c|c|}
\hline DMR Location & DMR Width & baseMean & $\log 2$ FoldChange & padj & Annotation & Nearest Gene \\
\hline chr10:1281019-1,282,852 & 1833 & 614.6687 & -1.39475 & 0.001099 & Intron & ADARB2 \\
\hline chr22:29515430-29,517,126 & 1696 & 2125.015 & -0.7487 & 0.001099 & Intron & KREMEN1 \\
\hline chr2:60693762-60,695,701 & 1939 & 293.8868 & -0.96653 & 0.003059 & Intron & BCL11A \\
\hline chr1:16888159-16,896,002 & 7843 & 1691.305 & 0.664717 & 0.018052 & $3^{\prime}$ UTR & MIR3675 \\
\hline chr15:22741828-22,744,210 & 2382 & 1025.047 & -1.02204 & 0.018052 & Exon & GOLGA6L1 \\
\hline chr15:32781660-32,783,301 & 1641 & 391.2485 & -0.56857 & 0.018052 & Distal Intergenic & GOLGA8O \\
\hline chr17:21901995-21,907,966 & 5971 & 1435.135 & 1.262002 & 0.018052 & Promoter $(<=1 \mathrm{~kb})$ & FLJ36000 \\
\hline chr19:24622360-24,624,613 & 2253 & 338.0393 & 1.234145 & 0.018052 & Distal Intergenic & HAVCR1P1 \\
\hline chr19:37783156-37,788,148 & 4992 & 786.5645 & 1.768082 & 0.018052 & Distal Intergenic & HKR1 \\
\hline chr2:92280419-92,282,186 & 1767 & 1109.498 & 1.027544 & 0.018052 & Distal Intergenic & ACTR3BP2 \\
\hline chr20:20317316-20,318,796 & 1480 & 713.2818 & -0.66627 & 0.018052 & Intron & INSM1 \\
\hline chr9:73946028-73,947,394 & 1366 & 290.2041 & -0.64868 & 0.024388 & Intron & TRPM3 \\
\hline chr18:15404549-15,410,901 & 6352 & 972.9929 & 1.687868 & 0.02956 & Distal Intergenic & LOC644669 \\
\hline chr2:92289472-92,292,822 & 3350 & 4738.836 & 1.060257 & 0.032703 & Distal Intergenic & ACTR3BP2 \\
\hline chr1:16932177-16,936,537 & 4360 & 710.057 & 0.489202 & 0.048809 & $5^{\prime}$ UTR & NBPF1 \\
\hline chr2:90371419-90,374,495 & 3076 & 1492.051 & 1.021356 & 0.048809 & Intron & MIR4436A \\
\hline chr2:90374619-90,378,951 & 4332 & 2470.989 & 1.010006 & 0.048809 & Intron & MIR4436A \\
\hline chr2:91595932-91,600,986 & 5054 & 2660.109 & 0.993924 & 0.048809 & Distal Intergenic & LOC654342 \\
\hline chr5:180899895-180,903,257 & 3362 & 290.8312 & 0.778464 & 0.048809 & Distal Intergenic & OR4F16 \\
\hline chr7:158998336-159,000,338 & 2002 & 314.5755 & -0.72705 & 0.048809 & Distal Intergenic & VIPR2 \\
\hline chr8:43792848-43,795,213 & 2365 & 1959.954 & 1.112517 & 0.048809 & Distal Intergenic & POTEA \\
\hline
\end{tabular}




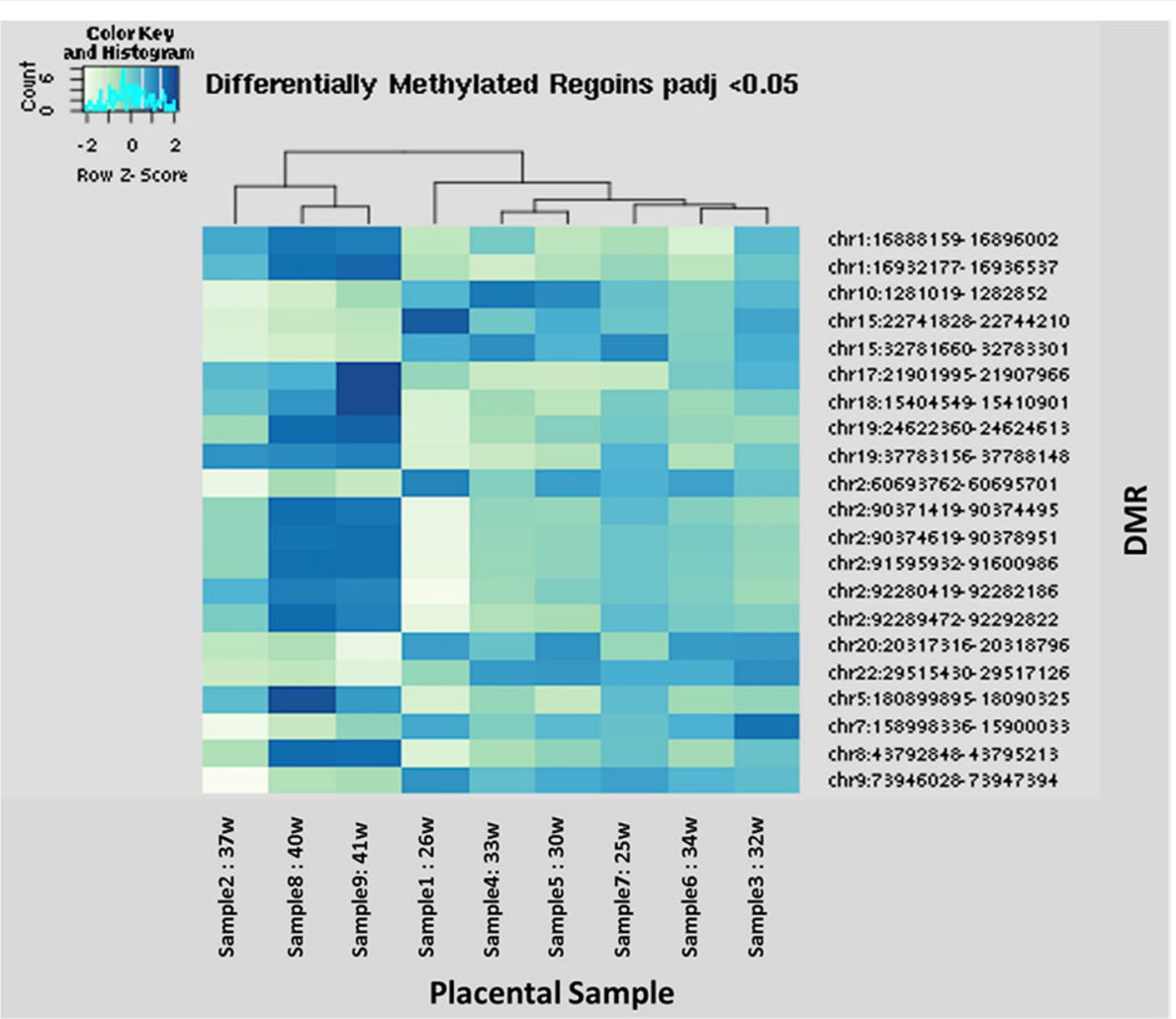

Fig. 1 Peak Intensity heat map: A heat map of the read counts of the 21 DMRs for each of the 9 samples. Samples 2, 8 and 9 are the term placentae, and the remaining is preterm. Unsupervised clustering was used to order the columns. Darker blue squares represent more reads/ higher methylation whereas lighter green squares represent less reads/lower methylation

have been shown to be associated with preterm birth and pregnancy. We compared the genes nearest to the DMRs associated with PTB and gestational age to transcription profiles from preterm and term placenta samples [20]. The results, shown in Tables 4 and 5, are for genes that were upregulated and downregulated, respectively. This table also shows the genes nearest DMRs that are contained within a set of genes that are in networks and pathways related to preterm birth, outlined in the Database for Preterm Birth (dbPTB) [21]. Lastly, we

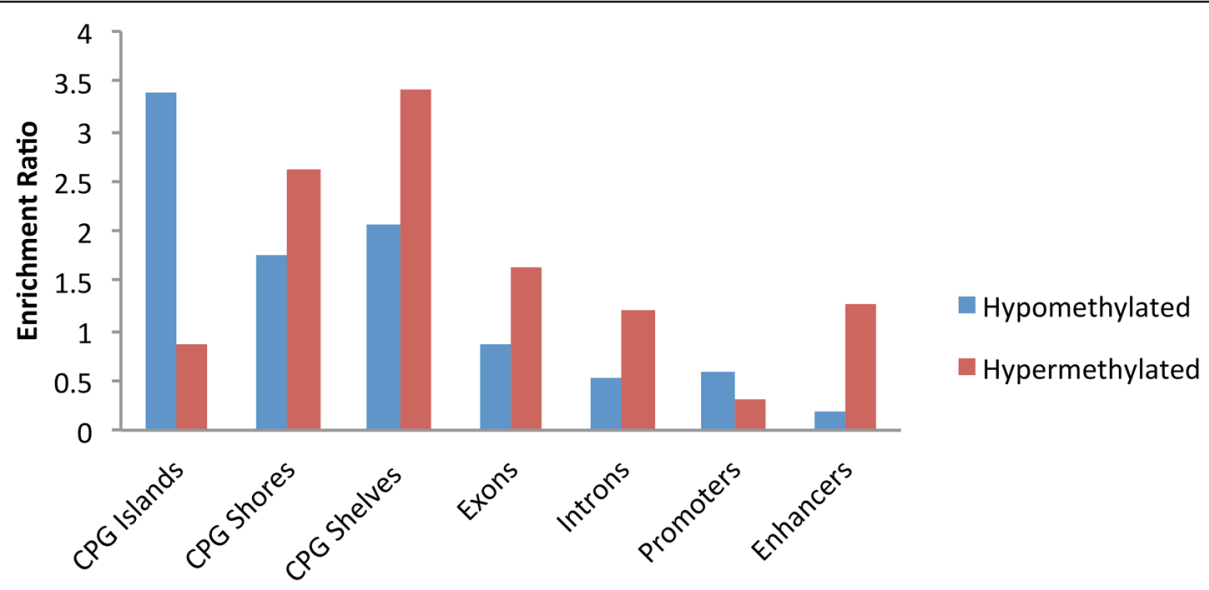

Genomic Features

Fig. 2 Enrichment of genomic features amongst differentially methylated regions: Genomic feature enrichment for hyper-methylated (left) and hypo-methylated (right) DMRs. Introns, Exons and CpG islands were obtained from UCSC Genome Browser and shores and shelves are defined as $2 \mathrm{~kb}$ and $4 \mathrm{~kb}$ up and down stream of the islands. Promoters and Enhancers were obtained from Roadmap Epigenome Placental cell line 
Table 3 Annotated DMR's Associated with Gestational Age

\begin{tabular}{|c|c|c|c|c|c|c|}
\hline DMR Location & DMR Width & baseMean & log2FoldChange & padj & Annotation & Nearest Gene \\
\hline chr11:1069274-1,070,940 & 1666 & 275.32 & -0.077895692 & 0.000669 & Distal Intergenic & MUC2 \\
\hline chr7:158509261-158,511,170 & 1909 & 785.4202 & -0.055129353 & 0.001379 & Distal Intergenic & NCAPG2 \\
\hline chr8:143302532-143,305,491 & 2959 & 708.6988 & -0.057666861 & 0.001379 & Intron & LINC00051 \\
\hline chr11:51578780-51,581,392 & 2612 & 5862.829 & 0.12089555 & 0.007632 & Distal Intergenic & OR4C46 \\
\hline chr15:22741828-22,744,210 & 2382 & 1025.047 & -0.091557436 & 0.007632 & Exon & GOLGA6L1 \\
\hline chr17:21901995-21,907,966 & 5971 & 1435.135 & 0.114589697 & 0.008451 & Promoter $(<=1 \mathrm{~kb})$ & FLJ36000 \\
\hline chr11:51587551-51,593,541 & 5990 & 6613.759 & 0.114797282 & 0.012877 & Distal Intergenic & OR4C46 \\
\hline chr10:115540476-115,542,108 & 1632 & 369.5293 & -0.058238042 & 0.015459 & Promoter (2-3 kb) & MIR4483 \\
\hline chr4:9875-10,674 & 799 & 300.733 & -0.066430834 & 0.020835 & Distal Intergenic & ZNF595 \\
\hline chr5:49415136-49,417,649 & 2513 & 629.6273 & 0.098693759 & 0.020835 & Distal Intergenic & EMB \\
\hline chr15:56071747-56,073,363 & 1616 & 280.2815 & -0.044766281 & 0.028409 & Distal Intergenic & PRTG \\
\hline chrY:59027085-59,033,404 & 6319 & 757.9891 & 0.085296918 & 0.028937 & Distal Intergenic & SPRY3 \\
\hline chr10:127579482-127,584,720 & 5238 & 732.2817 & 0.072848407 & 0.032657 & Promoter $(<=1 \mathrm{~kb})$ & DHX32 \\
\hline chr10:134878560-134,880,844 & 2284 & 289.1369 & -0.080610086 & 0.032657 & Distal Intergenic & ADGRA1 \\
\hline chr11:1795935-1,798,177 & 2242 & 452.7534 & -0.059025405 & 0.032657 & Distal Intergenic & MOB2 \\
\hline chr11:51570928-51,573,041 & 2113 & 623.7097 & 0.10472301 & 0.032657 & Distal Intergenic & OR4C46 \\
\hline chr11:51581774-51,585,209 & 3435 & 3634.642 & 0.099090126 & 0.032657 & Distal Intergenic & OR4C46 \\
\hline chr19:24622360-24,624,613 & 2253 & 338.0393 & 0.100239042 & 0.032657 & Distal Intergenic & HAVCR1P1 \\
\hline chr2:60693762-60,695,701 & 1939 & 293.8868 & -0.074300347 & 0.032657 & Intron & BCL11A \\
\hline chr2:90374619-90,378,951 & 4332 & 2470.989 & 0.08904097 & 0.032657 & Intron & MIR4436A \\
\hline chr2:92280419-92,282,186 & 1767 & 1109.498 & 0.085845619 & 0.032657 & Distal Intergenic & ACTR3BP2 \\
\hline chr21:11121793-11,128,301 & 6508 & 1510.744 & 0.043743747 & 0.032657 & Distal Intergenic & BAGE \\
\hline chr5:49413369-49,415,026 & 1657 & 555.3467 & 0.102896519 & 0.032657 & Distal Intergenic & $\mathrm{EMB}$ \\
\hline chr6:58775746-58,780,286 & 4540 & $59,219.18$ & 0.088992793 & 0.032657 & Distal Intergenic & GUSBP4 \\
\hline chr6:132921367-132,922,856 & 1489 & 702.8588 & -0.077101273 & 0.032657 & Distal Intergenic & TAAR3 \\
\hline chr7:155125413-155,128,850 & 3437 & 1162.764 & -0.038748136 & 0.032657 & Distal Intergenic & INSIG1 \\
\hline chr7:155199140-155,201,882 & 2742 & 355.5753 & -0.077494132 & 0.032657 & Distal Intergenic & EN2 \\
\hline chr2:91603906-91,606,341 & 2435 & 1002.445 & 0.094259612 & 0.033077 & Distal Intergenic & LOC654342 \\
\hline chr1:16888159-16,896,002 & 7843 & 1691.305 & 0.055571393 & 0.035079 & 3' UTR & MIR3675 \\
\hline chr2:91595932-91,600,986 & 5054 & 2660.109 & 0.085847679 & 0.035079 & Distal Intergenic & LOC654342 \\
\hline chr5:49428377-49,432,607 & 4230 & 1837.6 & 0.094529868 & 0.035079 & Distal Intergenic & $\mathrm{EMB}$ \\
\hline chr5:49434812-49,441,568 & 6756 & 3582.595 & 0.092033374 & 0.035079 & Distal Intergenic & EMB \\
\hline chr8:143093456-143,095,020 & 1564 & 434.2594 & -0.048625159 & 0.037056 & Distal Intergenic & MIR4472-1 \\
\hline chr9:43157894-43,160,792 & 2898 & 440.6311 & -0.051599615 & 0.038038 & Distal Intergenic & LOC642929 \\
\hline chr3:196625149-196,626,329 & 1180 & 5459.852 & 0.091127805 & 0.03925 & Intron & SENP5 \\
\hline chr1:2775172-2,776,643 & 1471 & 300.3145 & -0.049869539 & 0.043507 & Distal Intergenic & TТC34 \\
\hline chr1:161411315-161,417,356 & 6041 & 980.0181 & 0.059054185 & 0.043507 & Exon & FCGR2A \\
\hline chr1:227165108-227,167,121 & 2013 & 321.0379 & -0.05472419 & 0.043507 & Promoter $(<=1 \mathrm{~kb})$ & ADCK3 \\
\hline chr10:42639382-42,642,799 & 3417 & 471.9378 & 0.053591881 & 0.043507 & Distal Intergenic & LOC441666 \\
\hline chr12:117759233-117,761,187 & 1954 & 733.3737 & -0.051097394 & 0.043507 & Intron & NOS1 \\
\hline chr12:131743021-131,745,096 & 2075 & 402.1728 & -0.04241032 & 0.043507 & Distal Intergenic & LINC01257 \\
\hline chr14:104680716-104,682,479 & 1763 & 393.9317 & -0.039036454 & 0.043507 & Distal Intergenic & $\mathrm{KIF} 26 \mathrm{~A}$ \\
\hline chr14:106130890-106,133,431 & 2541 & 327.5869 & -0.050328469 & 0.043507 & Intron & ELK2AP \\
\hline chr18:9876-11,028 & 1152 & 869.3626 & -0.058790034 & 0.043507 & Distal Intergenic & ROCK1P1 \\
\hline
\end{tabular}


Table 3 Annotated DMR's Associated with Gestational Age (Continued)

\begin{tabular}{|c|c|c|c|c|c|c|}
\hline DMR Location & DMR Width & baseMean & log2FoldChange & padj & Annotation & Nearest Gene \\
\hline chr2:90380982-90,382,232 & 1250 & 703.058 & 0.087608099 & 0.043507 & Intron & MIR4436A \\
\hline chr2:90390888-90,393,740 & 2852 & 1224.857 & 0.082639282 & 0.043507 & Intron & MIR4436A \\
\hline chr2:232245135-232,247,014 & 1879 & 684.5341 & -0.049273959 & 0.043507 & Distal Intergenic & B3GNT7 \\
\hline chr2:233878888-233,880,783 & 1895 & 458.8731 & -0.042533652 & 0.043507 & Promoter (<=1 kb) & NGEF \\
\hline chr21:47233703-47,236,436 & 2733 & 660.0114 & -0.045879449 & 0.043507 & Intron & LOC100129027 \\
\hline chr22:28043663-28,045,838 & 2175 & 319.4394 & -0.045382128 & 0.043507 & Distal Intergenic & MN1 \\
\hline chr3:185842547-185,844,972 & 2425 & 338.0063 & -0.040820001 & 0.043507 & Distal Intergenic & ETV5 \\
\hline chr4:3679282-3,681,125 & 1843 & 632.2126 & -0.039394589 & 0.043507 & Promoter $(<=1$ kb) & LOC100133461 \\
\hline chr5:171997237-171,998,674 & 1437 & 574.0105 & -0.040752286 & 0.043507 & Distal Intergenic & NEURL1B \\
\hline chr5:172145042-172,146,642 & 1600 & 283.15 & -0.039405643 & 0.043507 & Distal Intergenic & DUSP1 \\
\hline chr7:35083300-35,086,409 & 3109 & 667.0347 & -0.063938851 & 0.043507 & Exon & DPY19L1 \\
\hline chr8:27426562-27,428,394 & 1832 & 618.5586 & -0.046453345 & 0.043507 & Distal Intergenic & CLU \\
\hline chrX:148615982-148,617,887 & 1905 & 496.8346 & -0.071187463 & 0.043507 & Promoter $(<=1 \mathrm{~kb})$ & IDS \\
\hline chr14:773222208-77,324,017 & 1809 & 358.4726 & -0.036853715 & 0.043857 & Exon & LRRC74A \\
\hline chr14:94213175-94,214,970 & 1795 & 274.9046 & -0.044559973 & 0.043963 & Intron & PRIMA1 \\
\hline chr8:143824284-143,827,190 & 2906 & 872.6112 & -0.056328342 & 0.043963 & Promoter (<=1 kb) & SLURP1 \\
\hline chr2:92289472-92,292,822 & 3350 & 4738.836 & 0.085456146 & 0.04533 & Distal Intergenic & ACTR3BP2 \\
\hline chr1:15170988-15,172,589 & 1601 & 341.8562 & -0.043070132 & 0.045985 & Intron & KAZN \\
\hline chr1:22873178-22,875,162 & 1984 & 628.9255 & -0.048195935 & 0.045985 & Distal Intergenic & EPHA8 \\
\hline chr15:32781660-32,783,301 & 1641 & 391.2485 & -0.044570446 & 0.045985 & Distal Intergenic & GOLGA8O \\
\hline chr2:92294963-92,300,499 & 5536 & 4633.964 & 0.085605878 & 0.048908 & Distal Intergenic & ACTR3BP2 \\
\hline chr4:5852906-5,854,271 & 1365 & 315.8726 & -0.042689188 & 0.048908 & Exon & CRMP1 \\
\hline chr7:15223273-15,225,157 & 1884 & 555.7869 & -0.050035386 & 0.048908 & Distal Intergenic & DGKB \\
\hline
\end{tabular}

compared the genes nearest our DMRs to a set of genes that have been previously found by Lynch et al. to be uniquely expressed in the endometrium of placental mammals and shown to be important in the evolution of pregnancy [22]. While the number of DMR associated genes overlapping each of these preterm birth gene sets is greater than the number expected by chance, this comparison was not statistically significant.

Nonetheless, it is of interest that a hyper-methylated DMR associated with PTB is in nearest proximity to the TFRC gene (Transferrin Receptor 1), which is associated with prematurity in placental transcription profiles and evolutionarily conserved endometrial genes. TFRC is an essential protein for iron transfer across the placenta and changes in its expression have been associated with IUGR and preeclampsia. In addition, a hypo-methylated DMR is associated with gestation age in the MLB2 (mannose binding lectin) gene, which overlaps the latter two comparative gene sets. MLB2 codes for a protein which plays a role in fetal inflammatory response to infection and injury.

\section{Pathway analysis}

Given that PTB is not a monogenic disorder, we were interested in the pathways associated with the genes

Table 4 Comparative Analysis of Nearest Genes to DMRs associated with PTB

\begin{tabular}{|c|c|c|c|c|}
\hline $\begin{array}{l}\text { Overexpressed in PTB } \\
\text { Placenta [20] }\end{array}$ & $\begin{array}{l}\text { Under expressed in PTB } \\
\text { Placenta [20] }\end{array}$ & $\begin{array}{l}\text { Mammalian Gain of } \\
\text { Function [22] }\end{array}$ & $\begin{array}{l}\text { Mammalian Loss of } \\
\text { Function [22] }\end{array}$ & $\begin{array}{l}\text { dbPTB Curated Gene Set } \\
{[21]}\end{array}$ \\
\hline $\begin{array}{l}\text { TFRC } \\
\text { NBPF10 } \\
\text { JAM3 } \\
\text { ARHGEF7 } \\
\text { DAPK1 }\end{array}$ & $\begin{array}{l}\text { GUSBP1 } \\
\text { MFSD2A } \\
\text { PSPC1 }\end{array}$ & $\begin{array}{l}\text { KREMEN1 } \\
\text { TFRC } \\
\text { DPY19L1 } \\
\text { C1orf159 } \\
\text { K1F14 } \\
\text { NEDD4L } \\
\text { C1QTNF3 } \\
\text { EDIL3 } \\
\text { PSD3 } \\
\text { ARPC5L }\end{array}$ & $\begin{array}{l}\text { C15orf27 } \\
\text { PRSS33 } \\
\text { CAPN13 } \\
\text { NPBWR2 } \\
\text { MYOM2 }\end{array}$ & $\begin{array}{l}\text { ATRNL1 } \\
\text { NOS1 }\end{array}$ \\
\hline
\end{tabular}


Table 5 Comparative Analysis of Nearest Genes to DMRs associated with Gestational Age

\begin{tabular}{|c|c|c|c|c|}
\hline $\begin{array}{l}\text { Overexpressed in PTB } \\
\text { Placenta [20] }\end{array}$ & $\begin{array}{l}\text { Under expressed in PTB } \\
\text { Placenta [20] }\end{array}$ & $\begin{array}{l}\text { Mammalian Gain of } \\
\text { Function [22] }\end{array}$ & $\begin{array}{l}\text { Mammalian Loss of } \\
\text { Function [22] }\end{array}$ & $\begin{array}{l}\text { dbPTB Curated Gene Set } \\
\text { [21] }\end{array}$ \\
\hline JAM3 & $\begin{array}{l}\text { PRKAG2 } \\
\text { MFSD2A } \\
\text { PSPC1 } \\
\text { BCL2 }\end{array}$ & $\begin{array}{l}\text { DPY19L1 } \\
\text { TMEM132C } \\
\text { C1orf159 } \\
\text { K1F14 } \\
\text { AKAP6 } \\
\text { ZNF532 } \\
\text { CMPK2 } \\
\text { SLC8A1 } \\
\text { ADCY2 } \\
\text { C1QTNF3 } \\
\text { KHDRBS3 }\end{array}$ & $\begin{array}{l}\text { FERMT1 } \\
\text { L1F1A } \\
\text { CAMK2A } \\
\text { KCNQ2 } \\
\text { RD3 } \\
\text { MBL2 } \\
\text { PRSS33 } \\
\text { GSG1L } \\
\text { EPHB1 } \\
\text { PDE6B } \\
\text { CLVS2 } \\
\text { MYOM2 }\end{array}$ & $\begin{array}{l}\text { CDC25A } \\
\text { COL1A2 } \\
\text { COL5A1 } \\
\text { ETV5 } \\
\text { F13A1 } \\
\text { GC } \\
\text { HS3ST3A1 } \\
\text { IL1R2 } \\
\text { KATNAL2 } \\
\text { LOXHD1 } \\
\text { MBL2 } \\
\text { MYH9 } \\
\text { NOS1 } \\
\text { SMAD6 } \\
\text { SOX17 } \\
\text { BEAN1 } \\
\text { KCNN3 }\end{array}$ \\
\hline
\end{tabular}

neared to the above DMRs. We reasoned this might provide insight into the functional context of the PTB associated DMRs. We found 9 canonical pathways significantly enriched in the PTB associated DMRs (-log $p$-value $>1.3)$ The most significant pathways included Superpathway of Citrulline Metabolism, Citrulline-Nitric Oxide Cycle, Fc-gamma Receptor Mediated Phagocytosis in Macrophages and the Urea Cycle. The Citrulline Metabolism pathway and the Citrulline Nitric Oxide Cycle pathway contain NOS1 (nitric oxide synthase 1) which has a proximal hyper-methylated DMR associated with both PTB and gestational age and also in the $\mathrm{dbPTB}$ gene set of networks and pathways related to PTB [21]. IPA also returned the top 25 gene interaction networks. The top scoring networks contained 25 genes from our DMR gene set and were related to cell death, organismal survival and gene expression.

We did additional pathway analysis on the set 177 unique genes nearest the 215 DMRs which were significant in both models and on the set of genes nearest the DMRs only significant in the PTB model. The aforementioned pathways remain the most significant ones. For the DMRs which were significant for both models, Superpathway of Citrulline Metabolism, Citrulline-Nitric Oxide Cycle, and the Urea Cycle were the top canonical pathways. For the DMRs only significant in the PTB model, Fc-gamma Receptor Mediated Phagocytosis in Macrophages remained in the top list of pathways, in addition to some new pathways: Integrin signaling, DNA damage signaling and FAK signaling.

\section{Discussion}

We used methylation-dependent immunoprecipitation followed by high throughput sequencing to generate non-biased, genome-wide map of DNA methylation in placenta from a wide range of gestational ages. We investigated regions for which there was differential methylation between preterm $(\leq 34$ weeks $)$ and term placentas ( $>37$ weeks), as well as regions for which the differences in methylation were associated with the continuous variable gestational age. Our results demonstrate significant differences in DNA methylation in preterm versus term placenta. Approximately half of the DMRs associated with preterm birth were not significantly associated with changes in gestational age. There were more hypo-methylated regions in preterm patients compared to term patients. The highest percentages of differentially methylated regions mapped to distal intergenic regions followed by introns, exons and then promoter regions. Mapping of these significant DMRs to the nearest genes demonstrated some overlap with patterns of differential gene expression in placentas from preterm and term patients [20]. There was also overlap with genes shown to be evolutionarily linked to preterm birth and to networks and pathways associated with preterm birth $[21,22]$.

Both candidate gene studies and genome-wide studies of DNA methylation in the placenta have been performed to investigate the mechanism(s) of preterm birth. One study found a positive association between global methylation and gestational age but others found little variation amongst the partially methylated domains across all three trimesters [11, 14]. Another study of promoter region methylation found overall differences in methylation between second and third trimester placentas, but not between first and second trimester [12]. Several studies examining gestational age and DNA methylation used umbilical cord blood to gain understanding into fetal programming and methylation state at birth. In one study, among the 39 genes showing differential methylation, 29 showed a decrease in methylation with increase in gestational age while the remainder showed an increase and no relationship to type of delivery [11]. Parets et al. studied methylation of cord blood 
leukocytes from 24 weeks to 41 weeks [10]. Most sites showed lower degrees of methylation with shorter gestational age, suggesting that one mechanism regulating the extent of methylation is gestational timing. We and others have also found associations with the preterm birth process itself. The Norwegian Mother and Child Cohort Study (MoBa) compared cord blood methylation with birthweight and found both increased and decreased patterns of methylation associated with specific genes [23]. Another study using the Illumina $450 \mathrm{k}$ array found 1400 variably-methylated regions which correlated with significant variables in the intrauterine environment including maternal smoking, maternal depression, maternal BMI, infant birthweight and gestational age [24]. Thus, while no unifying picture of the association between gestational age and DNA methylation has been demonstrated, we believe the mechanisms regulating the extent and pattern of placental DNA methylation include programmed changes linked to gestational timing as well as experiential changes. Our study, with a wide range of gestation ages, using a non-biased, genomewide approach, shows a significant effect of both gestational age as a continuous predictor and PTB status as a categorical predictor of placental DNA methylation.

The site of methylation may be crucial to the effect on gene expression or a reflection of the impact of environment on gene expression. Clusters of CpG's also known as $\mathrm{CpG}$ islands (CGI) are present in $5^{\prime}$ promoter regions of many genes. Methylation can also take place in shores and shelves, which are more distant to the promoter. Some studies have shown that tissue- and cancerspecific DMRs occur more frequently within CpG shores than CGIs themselves [25]. The functional implications of alterations in methylation are context-specific. Methylation in the immediate vicinity of the transcription start site is believed to block initiation, whereas methylation in the gene body may stimulate transcription elongation and/or have an impact on splicing [25]. We saw the greatest degree of differential methylation (almost 60\%) in distal intergenic regions. Second greatest differential methylation was seen in introns other than the first intron. In addition, enrichment analysis showed that hypomethylated DMRs were enriched for CpG Islands, while hyper-methylated DMR were enriched for CpG shores and shelves (Fig. 2). The annotation results, along with the later enrichment results, are consistent with the results from previous studies suggesting methylation is more dynamic outside of $\mathrm{CpG}$ islands in promoter regions. The enrichment of $\mathrm{CpG}$ islands amongst the hypo-methylated DMRs could be linked to chromosomal instability and imprinting [26]. The implications of the intergenic and intragenic methylation, as well as in shores and shelves on preterm birth are significant, yet mechanistically still unclear.
The most significant pathway associated with the genes nearest to the 427 DMRs we observed was Citrulline-Nitric Oxide Cycle, which contains the NOS1 gene. Our results found a hypermethylated DMR associated with both PTB and gestational age proximal to NOS1. NO is secreted by placenta [27] and known to modulate both fetal and utero placental blood flow [28]. Bielecki et al. found a lower concentration of $\mathrm{NO}$ in a group of women with premature contractile activity in comparison with gestational age-matched healthy pregnant women [29]. In another study the amniotic fluid concentration of $\mathrm{NO}$ was significantly higher in patients with intra-amniotic infection compared to those without intra-amniotic infection [30]. A decrease in NO production may contribute to the initiation of labor and cervical ripening [31]. A study suggests that NO produced by the placenta could play role in maintaining uterine quiescence by paracrine effect [32]. These results suggest that increased methylation of NOS1 may play an important role in the production of $\mathrm{NO}$ and subsequently preterm birth.

Another significant pathway was Fc-gamma Receptor Mediated Phagocytosis in Macrophages. There is abundant evidence for Fc gamma R mediated transcytosis of IgG in the placenta. The transfer of IgG from mother to fetus begins around 13 weeks of gestation and the total IgG concentrations in newborns is directly related to length of gestation. Infants born preterm have substantially lower IgG levels than full-term babies [33]. We also identified a DMR whose nearest gene is mannose binding lectin (MBL2), which has previously been identified by pathway and network analysis to be related to preterm birth and evolutionarily associated as well [21, 22]. $M B L 2$, found in amniotic fluid, is a serum protein involved in the activation of the complement system of the innate immune system and plays a role in fetal inflammatory response to infection and injury [34, 35]. It activates complement system by binding to carbohydrates, present on a wide range of proteins [36]. Moreover, fetal $M B L 2$ haplotypes and in utero exposure to viral infection increases the risk of preterm birth [37].

When we compared our DMR results to data sets important in preterm birth, we identified a hypermethylated peak whose nearest gene is transferrin receptor $1, T F R C$. TFRC is expressed in the placenta and mediates cellular iron uptake. Iron deficiency during pregnancy increases the risk of preterm birth [38]. While TFRC was upregulated spontaneous preterm birth in the Chim et al. placental expression study, it was also upregulated in the Lynch evolution of mammalian pregnancy and found to be reduced placentas with intrauterine growth restriction and preeclampsia [39]. Because prematurity, IUGR and preeclampsia have different pathogenic etiologies, the results suggest the importance of 
further investigation of the epigenetic regulation of TFRC with respect to pregnancy related disorders.

The current study demonstrates the feasibility of sample collection, technical analysis and data processing. Potential limitations of the study are the relatively small sample size and the diversity of patients. Nonetheless, in order to clearly define an effect of prematurity, we purposefully collected placental samples from a wide range of gestational ages. There was some variation in the mothers' clinical features beyond prematurity that may have impacted DNA methylation. Nonetheless, these unbiased data provide a useful reference for future studies by us and others. In addition, we chose to study genome-wide methylation using MeDIP-Seq due to its feasibility and moderate expense as compared to other techniques such as Whole Genome Bisulfite Sequencing. The affinity-based approach coupled with deep sequencing has a resolution of $100-300 \mathrm{bp}$ and is cost effective when single-base resolution is not necessary [40, 41]. Previous research suggested that at $1 \mathrm{x}$ coverage, a majority of the methylated CpG can be studied [40]. It is important to note that MeDIP-seq, similar to restriction enzyme digestion approaches, can only measure relative enrichment of methylated DNA rather than absolute methylation levels. Lastly, another advantage of MeDIPseq over WGBS is its ability to detect both 5Methylcytosine $(5 \mathrm{mC})$ and 5-hydroxymethylctyosine $(5 \mathrm{hmC})$ independently $[40,41]$.

\section{Conclusions}

We identified associations between DNA methylation and preterm birth, building on recent findings that prenatal environmental exposures mediate developmental programming effects through epigenetic changes [3, 42]. Our data demonstrate that in future studies it will be important to include gestational age matched samples with prenatal conditions like intrauterine growth restriction and environmental exposures such as drug use, environmental toxins and intrauterine infection. This will allow us to predict which local differences in methylation segregate with which combinations of phenotype. In addition, future studies should compare gestational age matched placentas from births due elective cesarean (before the onset of labor). These studies form the basis for future studies on the epigenetics of preterm birth, "fetal programming" and the impact of environment exposures on this important clinical challenge.

\section{Methods}

\section{Placental samples}

Placenta samples were collected by our research staff at Women \& Infants Hospital of Rhode Island. They obtained shortly after delivery from births ranging from 25 weeks to 41 weeks of gestational age. Samples of villous parenchyma were taken from four quadrants between the chorionic and basal plate. Care was taken to avoid maternal decidua and areas of hemorrhage or calcification. Samples were placed immediately into RNAlater ${ }^{T m}$ (Ambion, Inc., \#AM7021) and stored at $-80^{\circ} \mathrm{C}$ until DNA extraction. Preliminary studies have shown that macromolecules like RNA levels were similar from each sample site and that this approach was equal to or superior to immediate immersion in liquid nitrogen for prevention of RNA degradation [43, 44].

\section{DNA extraction}

Genomic DNA was extracted using the Qiagen DNeasy Blood and Tissue kit (Qiagen, \# 69506) and quantified on a NanoDrop 1000. $5 \mu \mathrm{g}$ of DNA was digested to fragment size 200-300 base pairs using dsDNA Fragmentase enzyme at $37^{\circ} \mathrm{C}$ for $30 \mathrm{~min}$ (New England Biolabs, \#MO348L). Fragments were end-repaired, 3 '-ends were adenylated, and appropriate adapter indexes were ligated using the Truseq protocol (Illumina). Between each reaction, fragments were cleaned using Agencourt AmPure magnetic beads (Beckman Coulter, \# A63881). Fragments were then amplified by $\mathrm{PCR}$ at $98^{\circ} \mathrm{C} / 30 \mathrm{~s} ; 10$ cycles of $98^{\circ} \mathrm{C} / 10 \mathrm{~s}, 60^{\circ} \mathrm{C} / 30 \mathrm{~s}, 72^{\circ} \mathrm{C} / 30 \mathrm{~s}$; and $72{ }^{\circ} \mathrm{C} 5 \mathrm{~min}$ with a hold at $10^{\circ} \mathrm{C}$. Enriched fragments were then cleaned using Agencourt AmPure magnetic beads and quantified before methylation-dependent immunoprecipitation.

\section{MeDIP-seq}

Methylated-DNA immunoprecipitation was performed using the Methylated-DNA IP kit (Zymo Research, \# D5101). $320 \mathrm{ng}$ of each sample was mixed with denaturation buffer and heated to $98^{\circ} \mathrm{C}$ for $5 \mathrm{~min}$. DNA is then mixed with MIB buffer, ZymoMag Protein A beads, and Mouse Anti-5-Methylcytosine from and incubated at $37^{\circ} \mathrm{C}$ for one hour, with mixing every $15 \mathrm{~min}$. The tubes were rocked, allowed to cluster, washed with reagent buffer and then eluted at $75^{\circ} \mathrm{C}$ for $5 \mathrm{~min}$. This was followed by a 2-min spin in a mini centrifuge at 18,000 g. The recovered DNA underwent $100 \mathrm{bp}$ paired-end sequencing in the Brown University Genomics Core in triplicate on an Illumina HiSeq 2500.

Raw sequence reads were separated according to sample-specific barcodes and mapped to the NCBI Build UCSC Hg19 human genome using the Burrows-Wheeler Aligner (BWA v0.6.2) [45]. The SAM files were converted to BAM files with SamTools (v0.1.18) [46] and duplicate reads (reads with the same start location) were removed using Picard Tools (v1.77) (https:/github.com/ broadinstitute/picard). We used Model-based Analysis for ChIP-Seq (MACS v1.4) [47] to identify significantly enriched regions (peaks) using $p<1 \times 10^{-5}$ as the significance threshold for each individual and technical replicate independently. 
Identification of differentially methylated regions

We used the R Bioconductor packages DiffBind (http:// bioconductor.org/packages/DiffBind/) and DESeq2 [48] to identify Differentially Methylated Regions (DMRs). We used DiffBind to identify a peak set for the study cohort, requiring that each individual's consensus peak set contain only peaks which were present in all three technical replicates. For each individual, the read count for each peak in the consensus peak set was merged by taking the sum over all three technical replicates. DMRs were identified using DESeq2. $P$-values were corrected using FDR with independent filtering of overall low mean counts.

\section{Genomic annotation and enrichment}

DMRs with a $p$-value $<0.01$ were annotated using $\mathrm{R}$ Bioconductor package ChIPseeker [19] to retrieve the nearest gene to the peaks of interest and annotate the genomic region of the peak. CpG islands and Refseq gene exons and introns were downloaded from the UCSC Genome Browser [49]. CpG shores and shelves were defined $2 \mathrm{~kb}$ and $4 \mathrm{~kb}$ up and downstream from the CpG islands, respectively. The Hg19 reference genome was spilt into $500 \mathrm{bp}$ windows and each window was annotated with the above genomic features if any overlap existed. The ChromHMM annotation of the Placenta Cell Line from the Roadmap Epigenome Project, obtained from the UCSC Genome Browser, was used to align the $500 \mathrm{bp}$ windows with "promoter" and "enhancer" state annotation [50]. The enrichment score for each genomic feature (CpG islands, shores, shelves, exons, introns, promoters, and enhancers) with respect to the DMRs was calculated via the method in Zhang et al. as the ratio between the fraction of DMRs overlapping widows with genomic feature and the fraction of total windows with the genomic feature [51].

\section{Comparative gene set analysis}

In order to examine the potential role of DNA methylation in the regulation of preterm birth we compared our DMRs with previously published gene sets associated with preterm birth and pregnancy.

Chim et al. used an array based approach to study differential placental gene expression between spontaneous preterm birth and spontaneous term birth. "They reported 240 significantly upregulated and 186 significantly downregulated genes in the placenta associated with spontaneous preterm birth." [20]. We also compared the significant DMRs with a gene set identified in curated articles, networks and pathways important in the risk of preterm birth [21]. This set was obtained via extensive literature curation and imputation. Lastly, we compared significant DMRs to a gene set linked evolutionarily to mammalian pregnancy [22]. In this work
Lynch et al. explore the evolution of pregnancy in placental mammals and identify 1532 gene that are uniquely expressed in the endometrium. Many of these genes were in close proximity to MER20, which regulate gene expression in response to progesterone and cAMP. These genes were broken down into gain and loss of expression in response to the stimuli.

\section{Pathway analysis}

Pathway analysis of the genes nearest to the DMRs with $p<0.01$ was performed using QIAGEN's Ingenuity Pathway Analysis (www.qiagen.com/ingenuity).

\section{Statistical analysis}

The Student's t-test was used to evaluate significant differences between cases and controls. A two- tailed $p<.05$ was considered to indicate statistical significant difference.

\section{Additional files}

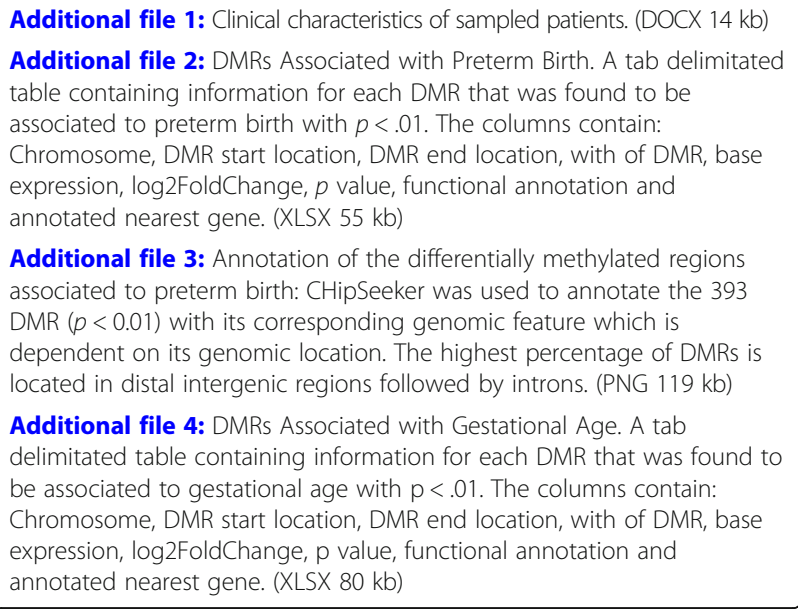
table containing information for each DMR that was found to be associated to preterm birth with $p<.01$. The columns contain: Chromosome, DMR start location, DMR end location, with of DMR, base expression, log2FoldChange, $p$ value, functional annotation and annotated nearest gene. (XLSX 55 kb)

Additional file 3: Annotation of the differentially methylated regions associated to preterm birth: CHipSeeker was used to annotate the 393 DMR $(p<0.01)$ with its corresponding genomic feature which is dependent on its genomic location. The highest percentage of DMRs is located in distal intergenic regions followed by introns. (PNG $119 \mathrm{~kb}$ )

Additional file 4: DMRs Associated with Gestational Age. A tab delimitated table containing information for each DMR that was found to be associated to gestational age with $p<.01$. The columns contain: Chromosome, DMR start location, DMR end location, with of DMR, base expression, log2FoldChange, $p$ value, functional annotation and annotated nearest gene. (XLSX $80 \mathrm{~kb}$ )

\section{Abbreviations}

BWA: Burrows-Wheeler Aligner; dbPTB: Database for Preterm Birth; DMR: Differentially methylated regions; DOHaD: Developmental Origins and Health and Disease; FDR: False discovery rate; GEO: Gene expression omnibus; IgG: Immunoglobulin G; IPA: Ingenuity Pathway Analysis; MACS: Model-based Analysis for ChIP-Seq; MeDIP-seq: Methylation dependent immunoprecipitation sequencing; NO: Nitric Oxide

\section{Acknowledgements}

We acknowledge the Kilguss Research Core at Women and Infant's Hospital of Rhode Island and the Genomics Core and the Center for Computation and Visualization at Brown University for their help in sample preparation, high-throughput sequencing and computational analysis.

\section{Authors' contributions}

The first author, JS, contributed to the design of this work, the analysis and interpretation of the data and wrote the first draft of this manuscript. AU contributed to the design, analysis and manuscript revision. JS and CS contributed to data acquisition. MM made substantial revisions to manuscript. JP contributed to the overall design, interpretation of the data and substantial manuscript revisions. Each author listed on the manuscript has read and approved the manuscript and takes full responsibility for its content. 


\section{Funding}

This work was supported by grants from the National Foundation March of Dimes \#21-FY14-154 which provided salary support for Drs. Uzun and Schuster as part of the Prematurity Initiative. Analytical work, including MEDIP sequencing, was carried out in the core research facilities supported by awards from the National Institutes of Health \#P30 GM114750, \#P20 RR18728 and \#P30 GM103410. Neither funding body contributed to the design, collection, analysis, interpretation nor writing of the manuscript.

\section{Availability of data and materials}

The dataset supporting the conclusions of this article is available in the GEO repository, under the following accession number: GSE120458 (https://www. ncbi.nlm.nih.gov/geo/query/acc.cgi?acc=GSE120458).

\section{Ethics approval and consent to participate}

Approved waiver for collection of de-identified clinical information and use of residual tissue, in this case placenta, was obtained from the Institutional Review Board at Women \& Infants Hospital of Rhode Island, Project No. 792532.

\section{Consent for publication}

The hospital admission consent includes approval for publication of deidentified research results. This was obtained from all subjects.

\section{Competing interests}

The authors declare no competing interests or conflicts of interest: No honorarium, grant, or other form of payment was given to anyone to produce the manuscript.

\section{Author details}

${ }^{1}$ Pediatrics, Women \& Infants Hospital, Providence, Rhode Island 02905, USA. ${ }^{2}$ Pediatrics, Center for Computational Molecular Biology, Brown Medical School, Brown University, Providence, Rhode Island 02906, USA. ${ }^{3}$ Molecular Biology, Cell Biology and Biochemistry, Brown University, Providence, Rhode Island 02906, USA. ${ }^{4}$ Pediatrics and Genetics, Hasbro Children's Hospital, Providence, Rhode Island 02905, USA. ${ }^{5}$ Providence, USA.

\section{Received: 19 December 2018 Accepted: 24 May 2019}

Published online: 28 June 2019

\section{References}

1. Muglia $\sqcup$, Katz M. The enigma of spontaneous preterm birth. N Engl J Med. 2010;362(6):529-35.

2. Kerkhof GF, Willemsen RH, Leunissen RW, Breukhoven PE, Hokken-Koelega AC. Health profile of young adults born preterm: negative effects of rapid weight gain in early life. J Clin Endocrinol Metab. 2012;97(12):4498-506.

3. Gluckman PD, Hanson MA, Cooper C, Thornburg KL. Effect of in utero and early-life conditions on adult health and disease. N Engl J Med. 2008;359(1): 61-73.

4. Menon R, Conneely KN, Smith AK. DNA methylation: an epigenetic risk factor in preterm birth. Reprod Sci. 2012;19(1):6-13.

5. Suter MA, Aagaard-Tillery KM. Environmental influences on epigenetic profiles. Semin Reprod Med. 2009;27(5):380-90.

6. Knight AK, Smith AK. Epigenetic Biomarkers of Preterm Birth and Its Risk Factors. Genes. 2016;7(4):15.

7. Ginder GD, Gnanapragasam MN, Mian OY. The role of the epigenetic signal, DNA methylation, in gene regulation during erythroid development. Curr Top Dev Biol. 2008;82:85-116.

8. Mikheev AM, Nabekura T, Kaddoumi A, Bammler TK, Govindarajan R, Hebert $M F$, et al. Profiling gene expression in human placentae of different gestational ages: an OPRU network and UW SCOR study. Reprod Sci. 2008; 15(9):866-77.

9. Winn VD, Haimov-Kochman R, Paquet AC, Yang YJ, Madhusudhan MS, Gormley M, et al. Gene expression profiling of the human maternal-fetal interface reveals dramatic changes between midgestation and term. Endocrinology. 2007;148(3):1059-79.

10. Parets SE, Conneely KN, Kilaru V, Fortunato SJ, Syed TA, Saade G, et al. Fetal DNA methylation associates with early spontaneous preterm birth and gestational age. PLoS One. 2013;8(6):e67489.
11. Schroeder JW, Conneely KN, Cubells JC, Kilaru V, Newport DJ, Knight BT, et al. Neonatal DNA methylation patterns associate with gestational age. Epigenetics. 2011;6(12):1498-504.

12. Novakovic B, Yuen RK, Gordon L, Penaherrera MS, Sharkey A, Moffett A, et al. Evidence for widespread changes in promoter methylation profile in human placenta in response to increasing gestational age and environmental/stochastic factors. BMC Genomics. 2011;12:529.

13. Maccani JZ, Koestler DC, Houseman EA, Marsit CJ, Kelsey KT. Placental DNA methylation alterations associated with maternal tobacco smoking at the RUNX3 gene are also associated with gestational age. Epigenomics. 2013; 5(6):619-30.

14. Chavan-Gautam P, Sundrani D, Pisal H, Nimbargi V, Mehendale S, Joshi S. Gestation-dependent changes in human placental global DNA methylation levels. Mol Reprod Dev. 2011;78(3):150.

15. Yuen RK, Penaherrera MS, von Dadelszen P, McFadden DE, Robinson WP. DNA methylation profiling of human placentas reveals promoter hypomethylation of multiple genes in early-onset preeclampsia. Eur J Hum Genet. 2010;18(9):1006-12.

16. Christensen BC, Houseman EA, Marsit CJ, Zheng S, Wrensch MR, Wiemels JL, et al. Aging and environmental exposures alter tissue-specific DNA methylation dependent upon CpG island context. PLoS Genet. 2009;5(8): e1000602.

17. Marsit CJ, Lambertini L, Maccani MA, Koestler DC, Houseman EA, Padbury $J F$, et al. Placenta-imprinted gene expression association of infant neurobehavior. J Pediatr. 2012;160(5):854-60 e2.

18. Zhao MT, Whyte JJ, Hopkins GM, Kirk MD, Prather RS. Methylated DNA immunoprecipitation and high-throughput sequencing (MeDIP-seq) using low amounts of genomic DNA. Cell Reprogram. 2014;16(3):175-84.

19. Yu G, Wang LG, He QY. ChIPseeker: an R/Bioconductor package for ChIP peak annotation, comparison and visualization. Bioinformatics. 2015;31(14): 2382-3

20. Chim SS, Lee WS, Ting YH, Chan OK, Lee SW, Leung TY. Systematic identification of spontaneous preterm birth-associated RNA transcripts in maternal plasma. PLoS One. 2012;7(4):e34328.

21. Uzun A, Dewan AT, Istrail S, Padbury JF. Pathway-based genetic analysis of preterm birth. Genomics. 2013;101(3):163-70.

22. Lynch VJ, Leclerc RD, May G, Wagner GP. Transposon-mediated rewiring of gene regulatory networks contributed to the evolution of pregnancy in mammals. Nat Genet. 2011;43(11):1154-9.

23. Engel SM, Joubert BR, Wu MC, Olshan AF, Haberg SE, Ueland PM, et al. Neonatal genome-wide methylation patterns in relation to birth weight in the Norwegian mother and child cohort. Am J Epidemiol. 2014;179(7):834-42.

24. Teh AL, Pan H, Chen L, Ong ML, Dogra S, Wong J, et al. The effect of genotype and in utero environment on interindividual variation in neonate DNA methylomes. Genome Res. 2014;24(7):1064-74.

25. Jones PA. Functions of DNA methylation: islands, start sites, gene bodies and beyond. Nat Rev Genet. 2012;13(7):484-92.

26. Daura-Oller E, Cabre M, Montero MA, Paternain $J$, Romeu A. Specific gene hypomethylation and cancer: new insights into coding region feature trends. Bioinformation. 2009;3(8):340-3.

27. Conrad KP, Joffe GM, Kruszyna H, Kruszyna R, Rochelle LG, Smith RP, et al. Identification of increased nitric oxide biosynthesis during pregnancy in rats. FASEB J. 1993;7(6):566-71.

28. Myatt $L$, Brewer A, Brockman DE. The action of nitric oxide in the perfused human fetal-placental circulation. Am J Obstet Gynecol. 1991;164(2):687-92.

29. Bielecki M, Tomasiak M, Jarocki S, Bodzenta-Lukaszyk A, Bielecki DA, Zdrodowska J. Nitric oxide in preterm labor. Ginekol Pol. 2003;74(5):339-44.

30. Hsu CD, Meaddough E, Aversa K, Hong SF, Lee IS, Bahodo-Singh RO, et al. Dual roles of amniotic fluid nitric oxide and prostaglandin E2 in preterm labor with intra-amniotic infection. Am J Perinatol. 1998;15(12):683-7.

31. Chwalisz K, Garfield RE. Role of nitric oxide in the uterus and cervix: implications for the management of labor. J Perinat Med. 1998;26(6):448-57.

32. Al-Hijji J, Andolf E, Laurini R, Batra S. Nitric oxide synthase activity in human trophoblast, term placenta and pregnant myometrium. Reprod Biol Endocrinol. 2003;1:51.

33. Saji F, Koyama M, Matsuzaki N. Current topic: human placental fc receptors. Placenta. 1994;15(5):453-66.

34. Ozdemir O, Dinleyici EC, Tekin N, Colak O, Aksit MA. Low-mannose-binding lectin levels in susceptibility to neonatal sepsis in preterm neonates with fetal inflammatory response syndrome. J Matern Fetal Neonatal Med. 2010; 23(9):1009-13. 
35. Malhotra R, Willis AC, Lopez Bernal A, Thiel S, Sim RB. Mannan-binding protein levels in human amniotic fluid during gestation and its interaction with collectin receptor from amnion cells. Immunology. 1994;82(3):439-44.

36. Petersen SV, Thiel S, Jensen L, Vorup-Jensen T, Koch C, Jensenius JC. Control of the classical and the MBL pathway of complement activation. Mol Immunol. 2000;37(14):803-11.

37. Gibson CS, Maclennan AH, Haan EA, Priest K, Dekker GA. Fetal MBL2 haplotypes combined with viral exposure are associated with adverse pregnancy outcomes. J Matern Fetal Neonatal Med. 2011;24(6):847-54

38. Allen LH. Anemia and iron deficiency: effects on pregnancy outcome. Am J Clin Nutr. 2000;71(5 Suppl):1280S-4S.

39. Mando C, Tabano S, Colapietro P, Pileri P, Colleoni F, Avagliano L, et al. Transferrin receptor gene and protein expression and localization in human IUGR and normal term placentas. Placenta. 2011;32(1):44-50.

40. Yong WS, Hsu FM, Chen PY. Profiling genome-wide DNA methylation. Epigenetics Chromatin. 2016;9:26.

41. Clark C, Palta P, Joyce CJ, Scott C, Grundberg E, Deloukas P, et al. A comparison of the whole genome approach of MeDIP-seq to the targeted approach of the Infinium HumanMethylation450 BeadChip((R)) for methylome profiling. PLoS One. 2012;7(11):e50233.

42. Wadhwa PD, Buss C, Entringer S, Swanson JM. Developmental origins of health and disease: brief history of the approach and current focus on epigenetic mechanisms. Semin Reprod Med. 2009;27(5):358-68.

43. Ponder KL, Salisbury A, McGonnigal B, Laliberte A, Lester B, Padbury JF. Maternal depression and anxiety are associated with altered gene expression in the human placenta without modification by antidepressant use: implications for fetal programming. Dev Psychobiol. 2011;53(7):711-23.

44. Wolfe LM, Thiagarajan RD, Boscolo F, Tache V, Coleman RL, Kim J, et al. Banking placental tissue: an optimized collection procedure for genomewide analysis of nucleic acids. Placenta. 2014;35(8):645-54.

45. Li H, Durbin R. Fast and accurate short read alignment with burrowswheeler transform. Bioinformatics. 2009;25(14):1754-60.

46. Li H, Handsaker B, Wysoker A, Fennell T, Ruan J, Homer N, et al. The sequence alignment/map format and SAMtools. Bioinformatics. 2009;25(16): 2078-9.

47. Zhang Y, Liu T, Meyer CA, Eeckhoute J, Johnson DS, Bernstein BE, et al. Model-based analysis of ChIP-Seq (MACS). Genome Biol. 2008:9(9):R137.

48. Love Ml, Huber W, Anders S. Moderated estimation of fold change and dispersion for RNA-seq data with DESeq2. Genome Biol. 2014;15(12):550.

49. Meyer LR, Zweig AS, Hinrichs AS, Karolchik D, Kuhn RM, Wong M, et al. The UCSC genome browser database: extensions and updates 2013. Nucleic Acids Res. 2013;41(Database issue):D64-9.

50. Roadmap Epigenomics C, Kundaje A, Meuleman W, Ernst J, Bilenky M, Yen $A$, et al. Integrative analysis of 111 reference human epigenomes. Nature. 2015;518(7539):317-30.

51. Zhang B, Xing X, Li J, Lowdon RF, Zhou Y, Lin N, et al. Comparative DNA methylome analysis of endometrial carcinoma reveals complex and distinct deregulation of cancer promoters and enhancers. BMC Genomics. 2014;15:868.

\section{Publisher's Note}

Springer Nature remains neutral with regard to jurisdictional claims in published maps and institutional affiliations.

Ready to submit your research? Choose BMC and benefit from:
- fast, convenient online submission
- thorough peer review by experienced researchers in your field
- rapid publication on acceptance
- support for research data, including large and complex data types
- gold Open Access which fosters wider collaboration and increased citations
- maximum visibility for your research: over 100M website views per year
At BMC, research is always in progress.
Learn more biomedcentral.com/submissions

